\title{
EFECTOS CLÍNICOS DE LOS PROBIÓTICOS: QUÉ DICE LA EVIDENCIA
}

\section{CLINICAL EFECTS OF PROBIOTICS: WHAT DOES THE EVIDENCE SAYS}

\author{
Claudia Manzano A., Diana Estupiñán G., Elpidia Poveda E. \\ Grupo de Investigación Nutrición y Salud, Instituto Alpina. \\ Alpina Productos Alimenticios S.A. Bogotá, Colombia.
}

\begin{abstract}
The scientific advances related to healthy properties of the probiotics have increased in a significant form. It is evident the positive effects on physiological functions. Considering the relevance in these advances, this review presents a summary of the scientific evidence supporting the principal biological effects of the probiotics in digestive function, systemic immune reply and lipid profile, pathologies relevant in the functional food development. Key words: Probiotics, clinical effects, digestive function, immune reply, lipid profile.
\end{abstract}

Este trabajo fue recibido el 10 de Diciembre de 2010, aceptado con modificaciones el 14 de Julio de 2011 y aceptado para ser publicado el 20 de Noviembre de 2011.

\section{INTRODUCCIÓN}

A comienzos del siglo XX el científico ElieMetchnikoff (1) postuló su hipótesis acerca de la influencia de la microbiota intestinal sobre el envejecimiento. Según él, los procesos de putrefacción en el intestino permitían la formación de toxinas que contribuían a la degeneración del cuerpo y propuso que el consumo de bacterias ácido lácticas en la leche fermentada podía disminuir los efectos adversos y reducir los procesos dañinos en el organismo (2).

En 1930, Minoru Shirota aisló de heces humanas una cepa de Lactobacillus casei que posteriormente cultivó en un medio lácteo para originar una bebida con probióticos (2). En 1965, Lilly y Stilwell utilizaron por primera vez el término probiótico para describir aquellas sustancias secretadas por un microorganismo que estimulan el crecimiento de otro, en contraposición al término antibiótico (3). Pero, fue en 1974 cuando Parker utilizó el término como hoy lo conocemos: organismos vivos que al ser ingeridos en cantidades adecuadas confieren un beneficio saludable en el huésped (4). Sin embargo, este concepto continua evolucionando, y actualmente la definición de probióticos es más amplia y estructurada.

Los probióticos son microorganismos vivos, principalmente bacterias, no patógenas, utilizados en forma de suplemento alimenticio, que tras ser ingeridos en cantidades suficientes, mejoran el equilibrio microbiano intestinal y provocan efectos benéficos sobre la salud de quienes los ingieren $(5,6)$.

Desde la década de los 80, la investigación científica sobre las propiedades saludables del consumo de probióticos ha aumentado considerablemente, lo cual ha promovido significativamente su uso. La presente revisión recoge de forma resumida evidencia científica sobre algunos de los efectos biológicos más estudiados en relación a los probióticos tales como diarreas agudas, efectos inmunomoduladores, alergias, síndrome de enfermedad inflamatoria intestinal, estreñimiento, síndrome de colon irritable, intolerancia a la lactosa y metabolismo lipídico. Estos efectos cobran cada vez mayor interés, debido a la repercusión que pueden tener en la salud de niños y adultos.

\section{Diarreas agudas}

En varios estudios de intervención controlados con placebo se ha evaluado la utilidad de los probióticos como tratamiento para la diarrea aguda, particularmente de tipo viral. Se ha demostrado que la administración de algunas cepas probióticas como Lactobacillus GG, L. reuteri, L. acidophilus y L.bulgaricus (sola o dentro 
de una terapia de rehidratación oral) se asocia a una disminución de la severidad y duración de las diarreas, tanto en poblaciones de países desarrollados como subdesarrollados $(6,7)$. De hecho, algunos meta-análisis han concluido que la terapia con probióticos puede reducir la duración de la enfermedad diarreica aguda aproximadamente en un día (8 - 11).

Para el caso del L. GG, algunos estudios muestran que disminuye en un día la duración de la diarrea aunque no modifica el número de deposiciones. También previene la diarrea asociada a los antibióticos, la diarrea nosocomial y la diarrea asociada a malnutrición pero no tiene efectos significativos cuando se trata de diarreas severas. Otras de las cepas evaluadas en el tratamiento y prevención de la diarrea son las especies de bifidobacterias, como el Bifidobacterium infantis y el B. bifidum en combinación con lactobacilos, mostrando de igual forma buenos resultados $(12-17)$.

En una revisión sistemática donde se analizaron los efectos de los probióticos para el tratamiento de la diarrea aguda se concluyó al igual que en otros estudios, que la administración de probióticos en conjunto con la terapia de rehidratación, resulta en una reducción de la duración y severidad de la diarrea, sin embargo, existiendo una marcada variabilidad clínica entre los diferentes resultados de los estudios y requieriendo más investigaciones para evaluar el efecto de manera cepa-específicas tanto en niños como en adultos; estos resultados permitirían a futuro desarrollar guías de tratamientos basados en la evidencia (11). Además, se menciona que las investigaciones deben tener en cuenta varios aspectos entre los que se encuentran: definición estándar para diarrea aguda, causas de la diarrea, si es de tipo viral o bacteriana, identificación segura del probiótico utilizado, confirmación de su viabilidad; así como identificar a través de investigaciones básicas los mecanismos de acción cepa específicos que permitan entender los aparentes beneficios de los probióticos en la diarrea aguda (11) .

En el tratamiento de la diarrea persistente en niños las investigaciones muestran que el uso de probióticos es prometedor como tratamiento coadyuvante porque disminuyen la duración y persistencia de la diarrea, sin embargo, la evidencia actual es insuficiente para recomendar su uso ya que los datos son inconclusos. En las mismas investigaciones se sugiere realizar estudios suficientemente fuertes y metodológicamente bien diseñados (18).

Con respecto a la prevención de la diarrea asociada a antibióticos se ha estudiado el papel de los Lactobacillus, concluyendo que la administración de esta cepa como un agente profiláctico durante el tratamiento antibiótico reduce el riesgo de desarrollar este tipo de diarrea en personas adultas pero no en pacientes pediátricos (19).

Algunos de los mecanismos que al parecer explican la acción de los probióticos en los episodios diarreicos son la producción de sustancias antimicrobianas (bacteriocinas, peróxido de hidrógeno y biosurfactantes) y la disminución del $\mathrm{pH}$ intestinal por el estímulo de organismos productores de ácido láctico, los cuales favorecen el crecimiento de organismos más beneficios. Otros probióticos aumentan la resistencia a la colonización al competir con los microorganismos patógenos por los sitios de unión en el endotelio intestinal y por los nutrientes que requieren para sobrevivir y desarrollarse. Sin embargo, se debe tener en cuenta que muchos modelos experimentales han revelado que los probióticos difieren mucho en sus mecanismos de acción, no solamente entre especies de probióticos, sino además entre ciertas cepas (20).

Se puede concluir que existe mejor evidencia científica del efecto benéfico de los probióticos en los procesos de diarrea aguda, y aunque existe evidencia del potencial efecto de ellos estos sobre la diarrea persistente y asociada a antibióticos, las metodologías utilizadas en los estudios no permiten afirmar que existe una evidencia contundente para afirmarlo.

\section{Efectos inmunomoduladores}

Se ha descrito que los probióticos pueden modular la respuesta inmune en animales y humanos no sólo a nivel de la mucosa intestinal, sino también a nivel sistémico. Dadas sus propiedades inmunomoduladoras, actualmente se evalúa la utilidad de los probióticos en el manejo preventivo o terapéutico de enfermedades inflamatorias. El consumo de probióticos podría tener un efecto positivo en la salud humana en algunas situaciones que pueden alterar el balance de la microbiota intestinal e influir en la respuesta inmune del individuo, tales como la alimentación con fórmulas infantiles, el tratamiento con antibióticos, los cambios fisiológicos relacionados con el envejecimiento, las enfermedades gastrointestinales y el estrés (21).

Los mecanismos de interacción de los probióticos con las células del sistema inmune son diversos. En el caso de las bacterias ácido lácticas, se ha observado que pueden ser captadas por las células M presentes en el epitelio y facilitar la estimulación del tejido linfoide asociado a la mucosa intestinal. Las células dendríticas pueden capturar bacterias probióticas al tener prolongaciones citoplasmáticas que pueden acceder al espacio luminal. Muchos de los efectos inducidos por los probióticos dependen de la interacción del microorganismo con la célula dendrítica, dada la capacidad que tiene de 
polarizar la respuesta inmunológica adaptativa. El efecto sobre la maduración de esta célula presentadora de antígeno y en su producción de citocinas depende de la cepa de probiótico, observándose casos de inhibición o estimulación de la producción de citocinas IL-10 e IL-12 lo cual, puede favorecer un estado de tolerancia o una respuesta inmune Th1, respectivamente (22).

Los efectos observados dependen del tipo celular estudiado. Mientras en células derivadas del bazo el Lactobacillus casei induce una fuerte respuesta Th1, valorada de acuerdo a los niveles de IL-12 producidos, en las placas de Peyer el efecto inductor por la producción de IL-12 es más bajo (23).

Las células epiteliales son estimuladas cuando interactúan con las bacterias probióticas. En un estudio reciente se observó que una mezcla de varias cepas de probióticos (VSL\#3) induce en las células epiteliales intestinales la producción de TNF- $\alpha$ y la activación de vías de señalización relacionadas con la activación de NFxB (24). Aunque aparentemente, estos probióticos están generando más inflamación, la estimulación de este tipo de respuesta innata se asocia a una inhibición del desarrollo de la enfermedad de Crohn, lo cual sugiere, que la inducción de una respuesta inmune equilibrada puede darse por mecanismos distintos a la inmunosupresión. Otros procesos inmunológicos que favorecen los probióticos son la producción de la inmunoglobulina A y M (25), la modulación de la producción de citocinas de la respuesta adaptativa, la liberación de quemoquinas, la activación de las células asesinas naturales y el desarrollo de células T reguladoras (26).

La modulación de la actividad fagocítica es otro de los efectos de los probióticos. Se ha documentado por una parte en voluntarios sanos, que la eliminación de alimentos fermentados en la dieta por dos semanas disminuye la actividad fagocítica de los leucocitos. Por otra parte el consumo de leche fermentada con L. acidophilus o L. johnsonii por tres semanas, aumenta la capacidad fagocítica. Del mismo modo, el consumo de L. gasseri y L. coryniformis por 2 semanas aumenta la actividad fagocítica de monocitos o neutrófilos en sujetos saludables. Al respecto, es importante tener en cuenta la dosis consumida porque es un factor determinante en los efectos deseados (27)[15].

La modulación del sistema inmune por parte de los probióticos puede tener beneficios para las personas en las diferentes etapas de la vida $(28,29)$. En niños con antecedentes familiares de eczema atópico se ha documentado el beneficio de los probióticos en la prevención de esta enfermedad, incluso desde el periodo de gestación através de la suplementación a la madre como posterior al nacimiento, suministrándolos por vía oral (28).
Existe evidencia del beneficio del tratamiento con bacterias ácido lácticas en niños, en pacientes desnutridos y adolescentes con diarrea. En cuanto a los jóvenes y adultos, se ha investigado la modulación del sistema inmune de bacterias ácido lácticas en personas sometidas a estrés, en prevención de infecciones del tracto urinario de mujeres en edad fértil y en el tratamiento de la alergia. Sin embargo, aún falta mayor investigación pues los estudios sobre estos efectos siguen siendo controvertidos y la evidencia existente no se considera concluyente (28). En el adulto mayor los estudios se han focalizado en observar la capacidad de los probióticos para contrarrestar la disminución del funcionamiento del sistema inmune que se presenta a esta edad (28).

En resumen, los diferentes estudios muestran la viabilidad de utilizar los probióticos para modular el sistema inmunológico, prevenir infecciones y controlar el proceso inflamatorio, pero los resultados son diversos, por lo cual, es necesario realizar investigaciones que disminuyan la brecha entre las diferencias que se encuentran con factores como: cepa o especie utilizada, dosis del probiótico, tiempo de suplementación y características de los sujetos estudiados (30). Lo anterior, permitirá realizar comparaciones y establecer conclusiones que beneficien el uso terapéutico y preventivo de los probióticos sobre el sistema inmune.

\section{Alergias}

En algunos estudios se han observado diferencias entre la composición de la flora microbiana de pacientes alérgicos e individuos sanos $(31,32)$. Por esto y dadas sus propiedades inmunomoduladoras, ha recibido considerable atención la posibilidad de utilizar los probióticos para la prevención y/o tratamiento de alergias (33).

Sin embargo, hasta el momento pocos estudios han encontrado resultados positivos sobre su uso terapéutico en pacientes alérgicos, especialmente a temprana edad (34-39). Un metaanálisis en el que se evalúo el efecto de los probióticos en la dermatitis atópica en niños, encontró un modesto efecto benéfico, sobre todo en pacientes con un nivel moderadamente severo de la enfermedad (40).

Es importante tener en cuenta que las diferencias que se han encontrado en los resultados dependen de varios factores como la cepa administrada, el momento de intervención terapéutica, la enfermedad alérgica estudiada y las combinaciones con otros suplementos alimenticios (41).

Por tal razón, se continúan buscando estrategias terapéuticas con probióticos que puedan ofrecer resultados exitosos. En una publicación reciente de Van de Pol y col (2010), se demostró que un simbiótico, mezcla de la cepa Bifidobacterium breve M-16V con varios prebióti- 
cos, reduce significativamente la producción de citocinas Th2 y mejora el pico espiratorio forzado (PEF) después del reto alergénico en adultos asmáticos y alérgicos a los ácaros (42). Otros estudios mostraron que el consumo de diferentes cepas de probióticos mejora los síntomas de la enfermedad alérgica en personas con riesgo de dermatitis atópica (43-47).

Al contrario de lo que se ha observado en su uso terapéutico, los probióticos han mostrado un mejor comportamiento en la prevención de las alergias (48) tal como lo sustentan modelos animales y un número importante de estudios clínicos (49-52). Una reciente revisión sistemática concluyó que éstos representan la intervención más prometedora para la prevención primaria de esta enfermedad (53).

Las investigaciones en gestantes han cobrado importancia debido a su potencial efecto en el sistema inmune del feto para la prevención futura de alergias. Los estudios indican que los probiótios pueden aumentar el potencial inmunológico de la leche materna y disminuir la sensibilización a alergias. La administración con Lactobacillus rhamnosus GG durante las cuatro semanas antes de dar a luz y en niños hasta de 3 meses después del nacimiento incrementó el potencial de inmunoprotección proporcionado por la leche materna, evaluado a través del incremento en la concentración del factor transformante de crecimiento $\beta 2$ presente en la leche y además disminuyó significativamente el riesgo de desarrollar eczema atópico comparado con hijos de madres del grupo control durante los primeros dos años de vida de los hijos (15\% y 47\%, respectivamente) (54). Otro estudio mostró que la suplementación con probióticos durante la gestación y durante la lactancia disminuía el riesgo de sensibilización a alergia en hijos de madres con dermatitis atópica (55). Otros estudios no muestran efectos positivos sobre la prevención de eczema atópico y la respuesta inmune del feto $(56,57)$.

El momento preciso para la intervención durante la gestación o en la lactancia no está totalmente claro aunque la mayoría de la evidencia indica que los mejores resultados preventivos se observan cuando se incluye la suplementación prenatal del probiótico, mientras que la administración después del nacimiento no muestra reducciones significativas del riesgo de alergias como el eczema (58).

Se puede deducir que los probióticos tienen efectos benéficos y muy potenciales en la prevención de alergias en niños con historia familiar y con alto riesgo de padecerlas, por lo cual, el consumo de probióticos desde la gestación y en el periodo de lactancia podría contribuir a disminuir los síntomas de estas alergias, sobre todo en niños, en los que la alergia es más severa.

\section{Enfermedad inflamatoria intestinal}

La colitis ulcerosa y la enfermedad de Crohn son dos formas de enfermedad inflamatoria intestinal, de las que se plantea que un desbalance entre las bacterias "protectoras" y patogénicas de la flora intestinal (disbiosis) puede estar relacionado a su patogénesis (59). El fundamento para utilizar probióticos en el tratamiento de estas enfermedades se basa en la posibilidad de colonizar el tracto intestinal con cepas bacterianas que promuevan una respuesta inmune de tipo protectora. En algunos estudios se ha encontrado que esta respuesta se basa en la producción de citocinas anti-inflamatorias, sin embargo, este no es el único mecanismo, varios estudios muestran que ciertas cepas probióticas inducen la producción de IL-12 a concentraciones bajas y en otros casos de células $\mathrm{T}$ reguladoras (26).

Aunque el tratamiento activo con probióticos induce cambios en la respuesta inmune de personas con enfermedad inflamatoria intestinal (caracterizadas por una disminución en el número de monocitos y células dendríticas productoras de IFN y un aumento de la secreción de IL-10 (60) y TGFb), la mayor parte de los ensayos clínicos realizados no han demostrado un efecto relevante en la evolución clínica de estos pacientes (61-63). No obstante, un reciente meta-análisis concluye que el tratamiento con probióticos puede ser efectivo para pacientes con colitis ulcerosa durante el periodo de remisión (64).

Una de las principales reflexiones sobre este efecto es que los probióticos podrían tener un beneficio en el manejo de la enfermedad inflamatoria intestinal, pero es necesario un mayor entendimiento del cambio de la microbiota intestinal en la patogénesis, lo cual facilitará el desarrollo ulterior de terapias microbiales efectivas basadas en probióticos.

\section{Estreñimiento}

Numerosos estudios demuestran que la adición de probióticos a bebidas lácteas puede contribuir a disminuir el estreñimiento, afección en la que el número de deposiciones es menor a tres veces por semana, asociado a heces duras o difíciles de evacuar, dolor y distención abdominal (65). Las investigaciones muestran que el beneficio sobre el estreñimiento puede ser provisto por distintas cepas, sin embargo, se observan diferencias en la población en la cual logran el efecto (favorable para niños y/o adultos) así como, en el indicador de cambio a nivel digestivo (número de deposiciones, consistencia de heces, dolor, entre otras). A continuación se mencionan algunas de las cepas probióticas para las cuales se han evidenciado resultados favorables sobre la constipación:

La administración de L. reuteri (DSM 17938) en ni- 
ños con estreñimiento crónico muestra efectos positivos en la frecuencia de la evacuación intestinal, aun cuando no se han observado efectos favorables en la consistencia de las heces (68). También, se ha comprobado que el L. casei rhamnosus Lcr35 y el Bifidobacterium breve, pueden ser benéficos $(67,68)$. El Bifidobacterium breve, incrementa la frecuencia de las evacuaciones, mejora la consistencia de las heces y disminuye el dolor abdominal en niños con estreñimiento funcional (69).

En adultos y niños la suplementación con los probióticos Bifidobacterium lactis DN-173010 ó Lactobacillus casei Shirota, ó Escherichia coli Nissle 1917 favorecen la frecuencia de las evacuaciones intestinales y la consistencia de las heces (68). Asimismo, se ha observado el efecto simbiótico del Lactobacillus acidophilus 145 y Bifidobacterium spp. 420 para prevenir la recurrencia de la enfermedad sintomática no complicada diverticular de colon, especialmente en aquellos pacientes con predominio de estreñimiento (69).

La mezcla de próbioticos, se considera una alternativa en el tratamiento del estreñimiento: en niños se ha demostrado que la combinación de los probióticos Bifidobacteria bifidum, B. infantis, B. longum, Lactobacilli casei, L. plantarum y L. rhamnosus genera una reducción significativa en los síntomas del esta patología, especialmente en relación al dolor abdominal y a la consistencia de heces (70). En adultos el consumo de yogur con una mezcla de probióticos Bifidobacterium breve, Bifidobacterium bifidum and Lactobacillus acidophilus ha demostrado un significativo aumento en la frecuencia de las evacuaciones (67).

El Bifidobacterium lactis Bb-12 es otra de las cepas probióticas cuyo consumo en bebidas lácteas fermentadas ha demostrado efectos benéficos a nivel intestinal. Específicamente, se relaciona con el aumento en el volumen de las heces y en la frecuencia de evacuación, así como, en una reducción significativa del hedor, y la aparición de formas más blandas de las mismas (71-73).

Los lácteos fermentados que contienen fibra dietaría tienen un efecto aditivo en el estreñimiento comparado con aquellos que sólo contienen el probiótico, especialmente, en personas donde el estreñimiento se relaciona a síndrome de colon irritable $(74,75)$.

En consecuencia, la evidencia científica actual muestra el beneficio de utilizar cepas probióticas en el manejo del estreñimiento, aún más si se adiciona fibra dietaría. Estas cepas pueden utilizarse a manera de suplementos o adicionadas en alimentos, esencialmente lácteos fermentados. No obstante, se considera importante realizar más investigaciones que permitan obtener una evidencia más sólida sobre el efecto en el estreñimiento. Estas investigaciones deben dirigirse a establecer por medio de estudios de intervención más representativos de la población la efectividad al ser administrados en una matriz alimentaria, la especificidad de las cepas sobre los síntomas del estreñimiento, la población en la cual pueden ser recomendados, la dosis asociada al efecto, y además, estudios donde se evalué el efecto aditivo al utilizar mezclas de probióticos y/o fibra dietaría.

\section{Síndrome de intestino irritable}

El síndrome de intestino irritable (SII) es una de las patologías gastrointestinales más comunes; se caracteriza por dolor abdominal, frecuencia de defecación alterada, consistencia de heces modificada, meteorismo e hinchazón (76). Diferentes estudios clínicos randomizados tanto en niños como en adultos han mostrado efectos significativos del consumo de probióticos sobre la frecuencia y severidad del dolor en el SII (77-83). Entre los probióticos que han sido estudiados se encuentran: Lactobacillus Lp299v, L. casei GG, L. acidophilus, Bifidobacterium animalis, B. lactis DN-173010, B. infantis 35624, y el grupo de probióticos VSL\#3.

Existe la hipótesis que relaciona la alteración de la composición bacteriana como base del SII, especialmente en aquellos pacientes cuya etiopatogenia provoca una alteración del equilibrio normal de la microbiota, como en la gastroenteritis bacterial aguda. El manejo con probióticos podría contribuir a restablecer este balance y a mejorar los síntomas especialmente la producción de gases (84).

Revisiones sistemáticas y meta-análisis han concluido que existe un potencial efecto benéfico de los probióticos en la disminución de la sintomatología general del SII, especialmente del dolor abdominal $(85,86)$. No obstante, los estudios presentan heterogeneidad clínica y estadística, con limitantes en el tipo y tamaño de población, dosis y tipo de probiótico utilizado, variación en la definición de los síntomas, corto tiempo de intervención, y limitaciones metodológicas, por lo que se ha recomendado realizar estudios clínicos randomizados aleatorizados de mayor duración y con un mayor rigor metodológico que demuestren el gran potencial de algunas cepas de probióticos $(87,88)$.

Se realizó un estudio en humanos para evaluar la cepa Bifidobacterium lactis HN019 y su potencial efecto sobre el tiempo de tránsito intestinal y síntomas digestivos frecuentes. El estudio encontró que el B Lactis HN019 disminuye el tiempo de tránsito intestinal y la severidad de los síntomas digestivos (estreñimiento, movimientos intestinales irregulares y flatulencia), de manera dosis dependiente, es decir, mayor efecto con la dosis más alta (89).

En resumen, los probióticos han mostrado un efecto 
benéfico en el SII sobre todo para controlar el dolor abdominal, pero son necesarios estudios con mayor rigor metodológico, para que los probióticos sean reconocidos como efectivos en el manejo del SII.

\section{Intolerancia a la lactosa}

La lactosa ingerida en los alimentos y no digerida en el intestino delgado pasa directamente al colon, produciendo los síntomas que caracterizan esté síndrome: dolor abdominal, diarrea, náuseas, flatulencia y/o distensión abdominal. Estos síntomas se pueden presentar en proporciones diferentes en cada persona ya que existe una variabilidad en la habilidad de la microbiota colónica para fermentar la lactosa; esta variabilidad explica los diferentes niveles de tolerancia en las personas (90).

El uso de probióticos en personas con intolerancia a la lactosa reduce los síntomas de inflamación o hinchazón, posiblemente como consecuencia de la presencia de la lactasa microbial presente en las bacterias acido lácticas, mejorando así la digestión de la lactosa (91-96). Sin embargo, existe una amplia variedad en la actividad de lactasa de los diferentes probióticos, lo cual, finalmente influye en su efecto (97).

La revisión de la literatura concluye que la evidencia permite llegar al consenso científico sobre los efectos benéficos de algunos probióticos para dos aplicaciones en particular: la intolerancia a la lactosa y la diarrea (92). Mientras que en otras revisiones se concluye que la suplementación con probióticos no alivia los síntomas y signos de intolerancia a la lactosa en adultos, excepto para controlar la flatulencia, sugiriendo que pueden ser efectivos para este síntoma $(98,99)$.

Por lo anterior, aunque existe evidencia en relación a que algunas cepas de probióticos pueden ser efectivas, se necesita mayor investigación, especialmente estudios clínicos con cepas específicas y metodologías objetivas.

\section{Metabolismo de los lípidos}

Existen estudios experimentales que demuestran un efecto benéfico del consumo de probióticos en el metabolismo de los lípidos (disminución de los niveles de colesterol y lipoproteínas de baja densidad, así como un aumento en los niveles de lipoproteínas de alta densidad) (100-104). Se han documentado estudios en humanos que evidencian un efecto benéfico sobre el nivel de lípidos tras el consumo de productos fermentados y adicionados con probióticos con combinación de cepas de lactobacilus y bifidobacterium (105-108).

Varias investigaciones no evidencian diferencias significativas entre el efecto del consumo de un yogur convencional y un yogur con probióticos (109-111), o en su defecto, sólo se han observado diferencias signi- ficativas en los niveles de colesterol en personas con hipercolesterolemia moderada (112).

Los mecanismos por los cuales los probióticos disminuyen el colesterol sérico no son claros, algunos estudios lo atribuyen a su habilidad de suprimir la reabsorción de ácidos biliares en la circulación enterohepatica y aumentar la excreción de esteroides ácidicos en heces (113-118). Se piensa que la disminución de colesterol en los medios de cultivo es secundaria a la precipitación del colesterol con ácidos biliares libres formados por las hidrolasas de las bacterias. También se ha observado que el consumo de probióticos disminuye en el hígado la enzima HMG- Coa reductasa que participa en la síntesis de colesterol, limitando la síntesis endógena de colesterol (119). Igualmente, los probióticos pueden unirse a la molécula de colesterol en el intestino delgado, habilidad que parece ser cepa específica (120), o tener la capacidad de convertir el colesterol a coprostanol en el intestino para ser excretado directamente en las heces, permitiendo por un proceso de homeostasis la reducción del colesterol sanguíneo (121).

Actualmente el nivel de evidencia no es contundente, incluso no se observan diferencias significativas entre el consumo de probióticos y bebidas lácteas fermentadas sobre el perfil lipídico, por lo cual es uno de los beneficios que más necesita estudios en humanos para corroborar el efecto, así como el mecanismo de acción.

\section{CONCLUSIÓN}

La evidencia científica muestra el potencial beneficio de los probióticos para prevenir o tratar algunas condiciones patológicas, así como para mejorar funciones fisiológicas. La evidencia es más fuerte en relación a su rol sobre la diarrea aguda, ayudando a disminuir su severidad y duración; en el SII, disminuyendo el dolor abdominal y síntomas gastrointestinales en general, y en el control de síntomas en niños con alergias de alta severidad.

Sus beneficios se pueden evidenciar en las diferentes etapas fisiológicas del ser humano y al parecer son cepa y dosis dependiente. Las diferencias halladas entre las cepas pueden ser de gran utilidad para obtener un beneficio potenciado sobre algunas condiciones o signos específicos de una patología o de un proceso fisiológico. Para profundizar en los beneficios y especificidad de las cepas se necesitan investigaciones que permitan validar los efectos, especialmente a nivel del metabolismo lipídico, inmunomodulación y en algunas patologías a nivel digestivo. Así mismo, profundizar en los mecanismos de acción, determinar la dosis a administrar, población objetivo y diferencias que pueden existir con la administración a través de un alimento o vía suplementos. 
La investigación biotecnológica y biomédica deberá mostrar paulatinamente el rol biológico de cada cepa lo que permitirá a futuro utilizarlas con mayor eficacia para la prevención y tratamiento clínico de diversas condiciones patológicas y del mejoramiento de diferentes funciones del organismo.

\section{RESUMEN}

Los avances científicos en relación a las propiedades saludables de los probióticos han aumentado significativamente, mostrando grandes avances en su efecto sobre diferentes funciones fisiológicas. Los hallazgos demuestran que las acciones a nivel fisiológico o clínico son cepa específicos, aspecto que toma cada día mayor relevancia en el desarrollo de alimentos funcionales asociados a probióticos. Teniendo en cuenta la importancia de estos avances, este artículo presenta de forma resumida evidencia que sustenta los principales efectos clínicos atribuidos a los probióticos en enfermedades relacionadas con el sistema digestivo, función inmune y metabolismo lipídico, patologías en las cuales el desarrollo de alimentos funcionales se ha venido enfocando.

Palabras claves: Probiótico, efectos clínicos, función digestiva, función inmune, metabolismo lipídico.

Dirigir la correspondencia a:

Profesora

Diana Consuelo Estupiñán González

Grupo de Investigación Nutrición y Salud

Instituto Alpina

Coordinadora Área Nutrición \& Salud

Alpina Productos Alimenticios S.A

Km 3 Via Sopo Briceño- Cundinamarca, Colombia

Teléfono: (57-1) 4238600 EXT 1564

FAX: (57-1) 4238630

E-mail: destupin@alpina.com.co

Agradecimientos: Agradecemos al profesor Luis Caraballo por la revisión y sugerencias a este manuscrito.

\section{BIBLIOGRAFÍA}

1. Metchnikoff . The prolongation of life. Dairy Sci 1907; 36:356. (Abstr)

2. Figueroa I, Gomez R L, Garcia GM. El beneficio de los probióticos. s.l. : Alfa editores técnicos 2006.

3. Amores R. Calvo A. Maestre D.Probióticos. Rev Española Quimiot 2004;17:131-9.

4. Dominguez-Bello MG, Blaser MJ. Do you have probioti in your future?. Microbes Infect 2008; 10:1072-6.

5. Isolauri E, Kirjavainen PV, Salminen S. Probiotics: a role in the treatment of intestinal infection and inflammation. Gut 2002; 50 (Suppl3):III54-9.

6. Young, R, Finocchiaro E, Sungsoo Cho JS. Prebiotics and Probiotics in Pediatric Diarrheal Disorders. En: Handbook of Prebiotics and Probiotics Ingredients. Health benefits and Food Applications. T New York: CRC Press, 2010.

7. Grandy G, Medina M, Soria R, Terán CG, Araya $M$. Probiotics in the treatment of acute rotavirus diarrhoea. A randomized, double-blind, controlled trial using two different probiotic preparations in Bolivian children. BMC Infect Dis 2010; 10:253.

8. Szajewska H, Mrukowicz JZ. 1. Probiotics in the treatment and prevention of acute infectious diarrhea in infants and children: a systematic review of published randomized, double-blind, placebocontrolled trials. J Pediatr Gastroenterol Nutr 2001 (33) Suppl 2:S17-25.

9. Van Niel, C. Lactobacillus Therapy for Acute Infectious Diarrhea in Children: A Meta-analysis. Pediatrics 2002; 109:4 678-68.

10. Huang JS, Bousvaros A, Lee JW, Diaz A, Davidson EJ. Efficacy of probiotic use in acute diarrhea in children: a meta-analysis. Dig Dis Sci 2002; 47(11):2625-34.

11. Allen SJ, Martinez EG, Gregorio GV, Dans LF. Probiotics for treating acute in fectious diarrhoea (Review). Cochrane Database Systematic Rev 2010; Issue 11.

12. Szajewska H, Kotowska M, Mrukowicz JZ, Armańska M, Mikołajczyk W. Efficacy of Lactobacillus GG in prevention of nosocomial diarrhea in infants. J Pediatr 2001; 138(3):361-5.

13. Saavedra J.M, Bauman N.A, PermanJ.A, Yolken R. H, OungI. Feeding of Bifidobacteriumbifidum and Streptococcus thermophilus to infants in hospital for prevention of diarrhoea and shedding of rotavirus. Lancet 1994;344:1046-49.

14. Arvola T, Laiho K, Torkkeli S, Mykkänen H, Salminen S, Maunula L, Isolauri E. Prophylactic Lactobacillus GG reduces antibiotic-associated diarrhea in children with respiratory infections: a randomized study. Pediatrics 1999; 104(5):e64.

15. Vanderhoof JA, Whitney DB, Antonson DL, Hanner TL, Lupo JV, Young RJ. Lactobacillus GG in the prevention of antibiotic-associated diarrhea in children. J Pediatr 1999; 135(5):564-8.

16. Basu S, Paul DK, Ganguly S, Chatterjee M, Chandra PK. Efficacy of high-dose Lactobacillus rhamnosus GG in controlling acute watery diarrhea in Indian children: a randomized controlled trial. J Clin Gastroenterol 2009; 43(3):208-13.

17. Oberhelman RA, Gilman RH, Sheen P, Taylor DN, 
Black RE, Cabrera L, et al. A placebo-controlled trial of Lactobacillus GG to prevent diarrhea in undernourished Peruvian children. J Pediatr 1999; 134(1):15-20.

18. Bernaola Aponte G, BadaMancilla CA, CarreazoPariasca NY, Rojas Galarza RA. Probiotics for treating persistent diarrhoeain children. Cochrane Database Systematic Rev 2010; Issue 11.

19. Kale-Pradhan PB, Jassal HK, Wilhelm SM. Role of Lactobacillus in the Prevention of AntibioticAssociated Diarrhea: A Meta-analysis. Pharmacoth 2010; 30(2):119-26.

20. O'Hara Ann M, Shanahan Fergus. Mechanisms of action of probiotics in intestinal diseases. Scientific World J 2007, 7: 31-46.

21. Nova E, Wärnberg J, Gómez-Martínez S, Díaz LE, Romeo J, Marcos A. Immunomodulatory effects of probiotics in different stages of life. Br J Nutr 2007; Suppl 1:S90-5.

22. Fink LN, Zeuthen LH, Christensen HR, Morandi B, Frøkiaer H, Ferlazzo G. Distinct gut derived lactic acid bacteria elicit divergent dendritic cellmediated NK cell responses. Int Immunol 2007; 12:1319-27.

23. Chiba Y, Shida K, Nagata S, Wada M, Bian L, Wang $\mathrm{C}$, et al. Well-controlled proinflammatory cytokine responses of Peyer's patch cells to probiotic Lactobacillus casei. Immunology 2010; 3:352-62.

24. Pagnini C, Saeed R, Bamias G, Arseneau KO, Pizarro TT, Cominelli F. Probiotics promote gut health through stimulation of epithelial innate immunity. Proc Natl Acad Sci 2010; 1:454-9.

25. Marteau P, Vaerman JP, Dehennin JP, Bord S, Brassart D, Pochart P, et al. Effects of intrajejunal perfusion and chronic ingestion of Lactobacillus johnsonii strain La1 on serum concentrations and jejunal secretions of immunoglobulins and serum protein in healthy humans. Gastroenterol Clin Biol 1997; 4:293-8.

26. Pronio A, Montesani C, Butteroni C, Vecchione S, Mumolo G, Vestri A, et al. Probiotic administration in patients with ileal pouch-anal anastomosis for ulcerative colitis is associated with expansion of mucosal regulatory cells. Inflamm Bowel Dis 2008; 5:662-8.

27. Yan, F y Polk, DB. Commensal bacteria in the gut: learning who our friends are. Curr Opin Gastroenterol 2004; 6: 565-71.

28. Romeo J, Nova E, Wärnberg J, Gómez-Martínez S, Díaz Ligia LE, Marcos A. Immunomodulatory effect of fibres, probiotics and synbiotics in different life-stages. Nutr Hosp 2010; 25:341-9.
29. Mitsuoka., Tomotari. Intestinal Flora and Aging Nutrition. Reviews. 1992;50 (Issue 12); 438-46.

30. Lomax AR, Calder PC. Probiotics, immune function, infection and inflammation: a review of the evidence from studies conducted in humans. Curr Pharm Des 2009; 15(13):1428-518.

31. Penders J, Thijs C, van den Brandt PA, Kummeling I, Snijders B, Stelma F, et al. Gutmicrobiota composition and development of atopic manifestations in infancy: the KOALA Birth Cohort Study Gut 2007; 5:661-7.

32. Watanabe S, Narisawa $\mathrm{Y}$, Arase S, Okamatsu H, Ikenaga T, Tajiri Y, et al. Differences in fecal microflora between patients with atopic dermatitis and healthy control subjects. J Allergy Clin Immunol 2003; 3:587-91.

33. Tang ML. Probiotics and prebiotics: immunological and clinical effects in allergic disease. Nestle Nutr Workshop Ser Pediatr Program, 2009; 64: 219-35; discussion 235-8, 251-7.

34. Weston S, Halbert A, Richmond P, Prescott SL. Effects of probiotics on atopic dermatitis: a randomised controlled trial. Arch Dis Child 2005; 9: 892-7.

35. Isolauri E, Arvola T, Sütas Y, Moilanen E, Salminen S. Probiotics in the management of atopic eczema. Clin Exp Allergy 2000; 11:1604-10.

36. Torii S, Torii A, Itoh $\mathrm{K}$, Urisu A, Terada A, Fujisawa $\mathrm{T}$, et al. Effects of oral administration of Lactobacillus acidophilus L-92 on the symptoms and serum markers of Lactobacillus acidophilus L-92 on the symptoms and serum markers of atopic dermatitis in children. Int Arch Allergy Immunol 2011; 154(3):236-45.

37. Woo SI, Kim JY, Lee YJ, Kim NS, Hahn YS. Effect of Lactobacillus sakei supplementation in children with atopic eczema-dermatitis syndrome. Ann Allergy Asthma Immunol 2010; 104(4):343-8.

38. Ouwehand AC, Nermes M, Collado MC, Rautonen $\mathrm{N}$, Salminen S, Isolauri E. Specific probiotics alleviate allergic rhinitis during the birch pollen season. World J Gastroenterol 2009; 15(26):3261-8.

39. Boyle RJ, Bath-Hextall FJ, Leonardi-Bee J, Murrell DF, Tang ML. Probiotics for treating eczema. Cochrane Database Syst Rev 2008; (4):CD006135.

40. Michail SK, Stolfi A, Johnson T, Onady GM. Efficacy of probiotics in the treatment of pediatric atopic dermatitis: a meta-analysis of randomized controlled trials. Ann Allergy Asthma Immunol 2008; 101(5):508-16.

41. Boyle RJ, Bath-Hextall FJ, Leonardi-Bee J, Murrell DF, Tang ML. Probiotics for the treatment of 
eczema: a systematic review. Clin Exp Allergy 2009; 39 (8):1117-27.

42. Van de Pol MA, Lutter R, Smids BS, Weersink EJ, van der Zee JS. Synbiotics reduce allergeninduced T-helper 2 response and improve peak expiratory flow in allergic asthmatics. Allergy 2011;66(1):39-47.

43. Morisset M, Aubert-Jacquin C, Soulaines P, Moneret-Vautrin DA, Dupont C. A non-hydrolyzed, fermented milk formula reduces digestive and respiratory events in infants at high risk of allergy. Eur J Clin Nutr 2011; 65(2):175-83.

44. Gerasimov SV, Vasjuta VV, Myhovych OO, Bondarchuk LI. Probiotic supplement reduces atopic dermatitis in preschool children: a randomized, double-blind, placebo-controlled, clinical trial. Am J Clin Dermatol 2010; 11(5):351-61.

45. Miniello VL, Brunetti L, Tesse R, Natile M, Armenio L, Francavilla R. Lactobacillus reuteri modulates cytokines production in exhaled breath condensate of children with atopic dermatitis. J Pediatr Gastroenterol Nutr 2010; 50(5):573-6.

46. Kim JY, Kwon JH, Ahn SH, Lee SI, Han YS, Choi YO, Lee SY, Ahn KM, Ji GE. Effect of probiotic mix (Bifidobacteriumbifidum, Bifidobacteriumlactis, Lactobacillus acidophilus) in the primary prevention of eczema: a double-blind, randomized, placebo-controlled trial. Pediatr Allergy Immunol 2010; 21(2 Pt 2):386-93.

47. Niers L, Martín R, Rijkers G, Sengers F, Timmerman H, Van Uden N, et al. The effects of selected probiotic strains on the development of eczema (the PandA study). Allergy; 2009; 64(9):1349-58.

48. Kopp MV, Salfeld P. Probiotics and prevention of allergic disease. Curr Opin Clin Nutr Metab Care 2009; 3:298-303.

49. Wickens K, Black PN, Stanley TV, Mitchell E, Fitzharris P, Tannock GW, et al. Probiotic Study Group. A differential effect of 2 probiotics in the prevention of eczema and atopy: a double-blind, randomized, placebo-controlled trial. J Allergy Clin Immunol 2008; 4:788-94.

50. Kalliomäki M, Salminen S, Poussa T, Arvilommi $\mathrm{H}$, Isolauri E. Probiotics and prevention of atopic disease: 4-year follow-up of a randomised placebocontrolled trial. Lancet 2003; 9372:1869-71.

51. Kukkonen K, Savilahti E, Haahtela T, JuntunenBackman K, Korpela R, et al. Probiotics and prebiotic galacto-oligosaccharides in the prevention of allergic diseases: a randomized, double-blind, placebo-controlled trial. J Allergy Clin Immunol 2007; 1:192.
52. Lodinová-Zádníková R, Prokesová L, Kocourková I, Hrdý J, Zizka J. Prevention of allergy in infants of allergic mothers by probiotic Escherichia coli. Int Arch Allergy Immunol 2010; 153(2):201-6.

53. Tang ML, Lahtinen SJ, Boyle RJ. Probiotics and prebiotics: clinical effects in allergic disease. Curr Opin Pediatr 2010; 22(5):626-34.

54. Tang, ML. Probiotics and prebiotics: immunological and clinical effects in allergic disease. Nestle Nutr Workshop Ser Pediatr Program 2009; 64: 219-35; discussion 235-8, 251-7.

55. Huurre A, Laitinen K, Rautava S, Korkeamäki M, Isolauri E. Impact of maternal atopy and probiotic supplementation during pregnancy on infant sensitization: a double-blind placebo-controlled study. Clin Exp Allergy 2008; 38(8):1342-8.

56. Boyle RJ, Ismail IH, Kivivuori S, Licciardi PV, Robins-Browne RM, Mah LJ, et al. Lactobacillus GG treatment during pregnancy for the prevention of eczema: a randomized controlled trial. Allergy 2011; 66(4):509-16.

57. Boyle RJ, Mah LJ, Chen A, Kivivuori S, RobinsBrowne RM, Tang ML. Effects of Lactobacillus GG treatment during pregnancy on the development of fetal antigen-specific immune responses. Clin Exp Allergy 2008; 38(12):1882-90.

58. ML,Tang. probiotics and prebiotics immunological and clinical effects in allergic disease. Nestle Nutr Workshop serPediatr program. 2009;64:219-35.

59. 59. CP Tamboli, CNeut, P Desreumaux, JF Colombel. Dysbiosis in inflammatory bowel disease. Gut 2004; 1:1-4.

60. Pathmakanthan S, Li CK, Cowie J, Hawkey CJ. Lactobacillusplantarum 299: beneficial in vitro immunomodulation in cells extracted from inflamed human colon. J Gastroenterol Hepatol 2004; 2:166-73.

61. Rahimi R, Nikfar S, Rahimi F, Elahi B, Derakhshani S, Vafaie M, et al. A meta-analysis on the efficacy of probiotics for maintenance of remission and prevention of clinical and endoscopic relapse in Crohn's disease. Dig Dis Sci 2008; 9: 2524-31.

62. Rolfe VE, Fortun PJ, Hawkey CJ, Bath-Hextall F. Probiotics for maintenance of remission in Crohn's disease. Cochrane Database Syst Rev 2006; 4: CD004826.

63. Shen J, Ran HZ, Yin MH, Zhou TX, XiaoDS. Meta-analysis: theeffect and adverse events of Lactobacilli versus placebo in maintenancetherapyfor Crohn disease. Intern Med J 2009; 39(2):103-9.

64. Sang LX, Chang B, Zhang WL, Wu XM, Li XH, Jiang M. Remission induction and maintenance effect 
of probiotics on ulcerative colitis: a meta-analysis . World J Gastroenterol 2010; 16(15):1908-15.

65. Camilleri, M. Disorders of gastrointestinal motility. [ed.] Ausiello D, eds In: Goldman L Philadelphia: Saunders Elsevier 2007; chap 138.

66. Coccorullo, P, HYPERLINK "http://www.ncbi. nlm.nih.gov/pubmed?term $=\% 22$ Coccorullo $\% 20$ P\% 22\%5BAuthor\%5D" Coccorullo P, HYPERLINK "http://www.ncbi.nlm.nih.gov/ pubmed?term $=\% 22$ Strisciuglio $\% 20 \mathrm{C} \% 22 \%$ 5BAuthor\%5D" Strisciuglio C, HYPELINK "http://www.ncbi.nlm.nih.gov/pubmed? term=\% 22Martinelli\%20M\%22\%5BAuthor\%5D" Martinelli M, HYPERLINK “http://www.ncbi. nlm.nih.gov/pubmed?term $=\% 22$ Miele $\% 20$ E\%22\%5BAuthor\%5D" Miele E, HYPELINK "http://www.ncbi.nlm.nih.gov/pubmed? term $=\% 22$ Greco $\% 20 \mathrm{~L} \% 22 \% 5 \mathrm{~B}$ Author\%5D" Greco L, HYPERLINK "http://www.ncbi.nlm.nih. gov/pubmed?term $=\% 22$ Staiano $\% 20 \mathrm{~A} \% 22 \% 5 \mathrm{~B}$ Author\%5D" et al. Lactobacillus reuteri (DSM 17938) in infants with functional chronic constipation: a double-blind, randomized, placebocontrolled study. J Pediatr 2010; 4(157): 598-602.

67. Tabbers, MM, de Milliano I, Roseboom MG, Benninga MA. Is Bifidobacterium breve effective in the treatment of childhood constipation? Results from a pilot study. Nutr J 2011; 23: 19.

68. Chmielewska, A y Szajewska, H. Systematic review of randomised controlled trials: probiotics for functional constipation. World J Gastroenterol 2010; 16: 69-75.

69. Lamiki, P, Tsuchiya J, Pathak S, Okura R, Solimene $\mathrm{U}$, Jain S, et al. Probiotics in diverticular disease of the colon: an open label study. J Gastrointestin Liver Dis 2010; 19:31-6.

70. Bekkali, NL, Bongers M EJ, Van den Berg MM, Liem O, Benninga MA. The role of a probiotics mixture in the treatment of childhood constipation: a pilot study. Nutr J [revista en Internet] 2007, [acceso 20 de octubre de 2011]; 6:17. Disponible en: HYPERLINK "http://www.nutritionj.com/ content/6/1/17" http://www.nutritionj.com/content/6/1/17 .

71. Shioya M, Nakaoka K, Igarashi R, Iizuka N, Abe T, Benno Y. Effect of fermented milk containing Bifidobacterium lactis FK 120 on the fecal flora and fecal properties in healthy females volunteers. JNutrit Food 2000; 3:7-18.

72. Matsumoto M, Imai $T$, Hironaka $T$, Kkume $H$, Watanabe M, Benno Y. Effect of yogurt with Bifidobacterium lactis LKM512 in improving fecal microflora and defecation of healthy volunteers. J Intest Microbiol 2001; 14, 97-102.

73. Gotteland M, Vizcarra M, Maury E. Efect of milk product containing probiotics and prebiotics on the digestive function of healthy and constipated subjects. Rev Chil Nutr 2010; 37 3: 340-51.

74. Choi SC, HYPERLINK "http://www.ncbi. nlm.nih.gov/pubmed?term $=\% 22 \mathrm{Kim} \% 20$ BJ\% 22\%5BAuthor\%5D" Kim BJ, HYPERLINK "http://www.ncbi.nlm.nih.gov/pubmed? term $=\% 22$ Rhee\% 20PL\%22\%5BAuthor\%5D" Rhee PL, HYPERLINK "http://www.ncbi. nlm.nih.gov/pubmed?term $=\% 22$ Chang $\% 20$ DK\%22\%5BAuthor\%5D" Chang DK, HYPERLINK "http://www.ncbi.nlm.nih.gov/pubmed?term $=\% 22 \mathrm{~S}$ on $\% 20 \mathrm{HJ} \% 22 \% 5 \mathrm{BAuth}$ or $\% 5 \mathrm{D}$ " Son HJ, HYPERLINK "http://www.ncbi. nlm.nih.gov/pubmed?term $=\% 22 \mathrm{Kim} \% 20$ JJ\%22\%5BAuthor\%5D" Kim JJ, et al. Probiotic fermented milk containing dietary fiber has additive effects in ibs with constipation compared to plain probiotic fermented milk. Gut Liver 2011; 5:22-8.

75. De Paula, JA, Carmuega, E y Weill, R. Effect of the ingestion of a symbiotic yogurt on the bowel habits of women with functional constipation. Acta Gastroenterol Latinoam 2008;38(1):16-25.

76. Association, American Gastroenterological. Technical review on irritable bowel syndrome. s.l.: Gastroenterology 2002; 123: 2018-31.

77. Nobaek S, Johansson ML, Molin G, Ahrne S, Jeppsson B. Alteration of intestinal microflora is associated with reduction in abdominal bloating and pain in patients with irritable bowel syndrome. . s.l. : Am J Gastroenterol 2000; 95: 1231-8.

78. Niedzielin K, Kordecki H, Birkenfeld B. A controlled, double-blind, randomized study on the efficacy of Lactobacillus plantarum 299V in patients with irritable bowel syndrome. Eur J Gastroenterol Hepatol 2001; 13:1143-7.

79. Sen S, Mullan MM, Parker TJ, Woolner JT, Tarry SA, Hunter JO. Effect of lactobacillus plantarum $299 \mathrm{v}$ on colonic fermentation and symptoms of irritable bowel syndrome. Dig Dis Sci., 2002; 47:2615-20.

80. Wochenschr, Dtsch Med. Probiotic therapy of the irritable bowel syndrome. Gastroenterol Hepatol (N Y) 2010; 6(1): 39-44. 2011; 136(8):371-5.

81. Francavilla R, Miniello V, Magistà AM, De Canio A, Bucci N, Gagliardi F, et al. A randomized controlled trial of Lactobacillus GG in children with functional abdominal pain. Pediatrics 2010;126 (6):1445-52 . 
82. Guandalini S, Magazzù G, Chiaro A, La Balestra V, Di Nardo G, Gopalan S, Sibal A, Canani RB, Lionetti P, Setty M. VSL\#3 improves symptoms in children with irritable bowel syndrome: a multicenter, randomized, placebo-controlled, doubleblind, crossover study. J Pediatr Gastroenterol Nutr 2010; 51(1):24-30.

83. Horvath A, Dziechciarz P, Szajewska H. Metaanalysis: Lactobacillus rhamnosus GG for abdominal pain-related functional gastrointestinal disorders in childhood. Aliment Pharmacol Ther 2011; 33(12):1302-10.

84. Kassinen A, HYPERLINK "http://www.ncbi. nlm.nih.gov/pubmed?term $=\% 22 \mathrm{Kassinen} \% 20$ A\%22\%5BAuthor\%5D” HYPELINK “http://www. ncbi.nlm.nih.gov/pubmed?term $=\% 22$ KrogiusKurikka\%20L\%22\%5BAuthor\%5D” KrogiusKurikkaL, HYPERLINK “http://www.ncbi.nlm.nih. gov/pubmed?term $=\% 22 \mathrm{M} \% \mathrm{C} 3 \%$ A4kivuokko $\% 20$ H\%22\%5BAuthor\%5D" Mäkivuokko H, HYPERLINK "http://www.ncbi.nlm.nih.gov/ pubmed?term $=\% 22$ Rinttil $\%$ C $3 \%$ A $4 \% 20$ T\%22\%5B Author\%5D" Rinttilä T, HYPERLINK "http://www.ncbi.nlm.nih.gov/pubmed? term $=\% 22$ Paulin $\% 20 \mathrm{~L} \% 22 \% 5 \mathrm{~B}$ Author\%5D" Paulin L, HYPERLINK "http://www.ncbi. nlm.nih.gov/pubmed?term $=\% 22$ Corander $\% 20$ J\%22\%5BAuthor\%5D" Corander J, et al. The fecal microbiota of irritable bowel syndrome, patients differs significantly from that of healthy subjects. Gastroenterology 2007; 133: 24-33.

85. Nourieh Hoveyda, Carl Heneghan1, Kamal R Mahtani, Rafael Perera, Nia Roberts and Paul Glasziou. A systematic review and meta-analysis: probiotics in the treatment of irritable bowel syndrome. World J Gastroenterol 2008; 14(17): 2650-61.

86. McFarland LV, Dublin S. Meta-analysis of probiotics for the treatment of irritable bowel syndrome. World J Gastroenterol 2008; 14: 2650-61.

87. McGuckin MA, Eri R, Simms LA, Florin T, Radford-Smith G. Intestinal barrier dysfunction in inflammatory bowel diseases. Inflamm Bowel Dis 2009; 15:100-13.

88. Moayyedi P, Ford HYPERLINK "http://gut.bmj. $\mathrm{com} / \mathrm{search}$ ?author $1=\mathrm{A}+\mathrm{C}+$ Ford\&sortspec $=$ date \&submit=Submit" A C, Talley NJ HYPERLINK "http://gut.bmj.com/search?author $1=\mathrm{N}+\mathrm{J}+$ Talle y\&sortspec $=$ date $\&$ submit $=$ Submit", Cremonini HYPERLINK "http://gut.bmj.com/search?author $1=\mathrm{F}+$ Cremonini\&sortspec $=$ date $\&$ submit $=$ Submit" F, Foxx-Orenstein HYPERLINK "http://gut.bmj. com/search?author $1=\mathrm{A}+\mathrm{E}+\mathrm{Foxx}$-Orenstein\&sort spec $=$ date \&submit=Submit" A E , HYPERLINK "http://gut.bmj.com/search?author1=L+J+Brandt\& sortspec $=$ date \&submit=Submit" L J Brandt, et al. The efficacy of probiotics in the therapy of irritable in the therapy of irritable bowel syndrome (IBS): a systematic review. Gut 2010; 59: 325-32.

89. Waller, PA, Gopal PK., Leyer GJ, Ouwehand AC, Reifer C, Stewart ME, et al. Dose-response effect of Bifidobacterium lactis HN019 on whole gut transit time and functional gastrointestinal symptoms in adults. Scand J Gastroenterol 2011; 46:1057-64.

90. Arola, H y Tamm, A. Metabolism oflactose in the human body. Scand J Gastroenterol Suppl 1994; 202: 21-5.

91. MacLean WC Jr, HYPERLINK "http://www. ncbi.nlm.nih.gov/pubmed?term $=\% 22$ Fink $\% 20$ BB\% 22\%5BAuthor\%5D" Fink BB, HYPERLINK "http://www.ncbi.nlm.nih.gov/pubmed?term= \%22Schoeller\%20DA\%22\%5BAuthor\%5D" Schoeller DA, HYPERLINK "http://www. ncbi.nlm.nih.gov/pubmed?term $=\% 22$ Wong $\% 20$ W\%22\%5BAuthor\%5D” Wong W, HYPERLINK "http://www.ncbi.nlm.nih.gov/pubmed?term= \%22Klein\%20PD\%22\%5BAuthor\%5D” Klein PD. Lactose assimilation by full-term infants: relation of and $\mathrm{H} 2$ breath tests with fecal excretion. Pediatr Res 1983; 17: 629-33.

92. Hove H, Norgaard H, Mortensen PB. Eur J Clin Nutr 1999; 53: 339-50.

93. Johnson, AO, y otros Adaptation of lactose maldigesters to continued milk intakes. Am J Clin Nutr 1993; 58: 879-81.

94. Florent C, HYPERLINK "http://www.ncbi. nlm.nih.gov/pubmed?term $=\% 22$ Flourie $\% 20$ B\%22\%5BAuthor\%5D" Flourie B, HYPERLINK "http://www.ncbi.nlm.nih.gov/pubmed?term= \%22Leblond\%20A\%22\%5BAuthor\%5D” Leblond A, HYPERLINK "http://www.ncbi.nlm. nih.gov/pubmed?term $=\% 22$ Rautureau $\% 20$ M\%22\%5BAuthor\%5D" Rautureau M, HYPERLINK "http://www.ncbi.nlm.nih.gov/pubmed? term $=\% 22$ Bernier\%20JJ\%22\%5B Author\%5D" Bernier JJ, HYPERLINK "http://www.ncbi. nlm.nih.gov/pubmed?term $=\% 22$ Rambaud $\% 20$ JC\%22\%5BAuthor\%5D" Rambaud JC. Influence of chronic lactulose ingestión on the colonic metabolism of lactulose in man (an in vivo study). J Clin Invest 1985; 75: 608-13.

95. Martini MC, Lerebours EC, Lin WJ, Harlander SK, Berrada NM, Antoine JM et al. Strains and species of lactic acid bacteria in fermented milks (yogurts): 
effect on in vivo lactose digestion. Am J Clin Nutr 1991; 54:1041-6.

96. De Vrese M, Stegelmann A, Richter B, Fenselau S, Laue C, Schrezenmeir J. Probiotics - compensation for lactase insufficiency. Am J Clin Nutr 2001; 73(2, Suppl)421S-9S.

97. HYPERLINK "http://www.ncbi.nlm.nih.gov/ pubmed?term $=\% 22 \mathrm{Vesa} \% 20 \mathrm{TH} \% 22 \% 5 \mathrm{BAuthor} \%$ 5D” Vesa TH, HYPERLINK “http://www.ncbi. nlm.nih.gov/pubmed?term $=\% 22$ Marteau $\% 20$ P\%22\%5BAuthor\%5D" Marteau P, HYPERLINK "http://www.ncbi.nlm.nih.gov/pubmed?term=\% 22Zidi\%20S\%22\%5BAuthor\%5D" Zidi S, HYPERLINK "http://www.ncbi.nlm.nih.gov/pubmed? term $=\% 22$ Briet $\% 20 \mathrm{~F} \% 22 \% 5 \mathrm{BAuthor} \% 5 \mathrm{D}$ " Briet F, HYPERLINK "http://www.ncbi.nlm. nih.gov/ pubmed?term $=\% 22$ Pochart $\% 20$ P\% 22\%5B Author\%5D" Pochart P, HYPERLINK "http://www.ncbi.nlm.nih.gov/pubmed? term $=\% 22$ Rambaud\%20JC\%22\%5BAuthor\%5D" Rambaud JC. Digestion and tolerance of lactose from yoghurt and different semi-solid fermented dairyproducts containing Lactobacillus acidophilusand bifidobacteria in lactosemaldigesters-is bacterial lactaseimportant. Eur J Clin Nutr 1996; 50: 730-3.

98. Levri KM, Ketvertis K, Deramo M, Merenstein JH, D'Amico F. Do probiotics reduce adult lactose intolerance? A systematic review. J Fam Pract 2005; 54:613-20.

99. Agency for Healthcare Research and Quality. Lactose Intolerance and Health. The Minnesota Evidence-based Practice Center 2010. No. 192.

100. Nguyen TD, Kang JH, Lee MS.Characterization of Lactobacillus plantarum $\mathrm{PH} 04$, a potential probiotic bacterium with cholesterol-lowering effects. Int J Food Microbiol 2007;113(3):358-61.

101. Fukushima M, Yamada A, Endo T, Nakano M. Effects of a mixture of organisms, Lactobacillus acidophilus or Streptococcus faecalis on delta6desaturase activity in the livers of rats fed a fatand cholesterol-enriched diet. Nutrition 1999; 15(5):373-8.

102. Taranto MP, Medici M, Perdigon G, Ruiz Holgado AP, Valdez GF. Effect of Lactobacillus reuteri on the prevention of hypercholesterolemia in mice. $\mathrm{J}$ Dairy Sci 2000; 83(3):401-3.

103. Wang Y, Xu N, Xi A, Ahmed Z, Zhang B, Bai X. Effects of Lactobacillus plantarum MA2 isolated from Tibet kefir on lipid metabolism and intestinal microflora of rats fed on high-cholesterol diet. Appl Microbiol Biotechnol 2009; 84(2):341-7.
104. Park YH, Kim JG, Shin YW, Kim SH, Whang KY. Effect of dietary inclusion of Lactobacillus acidophilus ATCC 43121 on cholesterol metabolism in rats. J Microbiol Biotechnol 2007; 17(4):655-62.

105. Sadrzadeh-Yeganeh H, Elmadfa I, Djazayery A, Jalali M, Heshmat R, Chamary M. The effects of probiotic and conventional yoghurt on lipid profile in women. Br J Nutr 2010; 103(12):1778-83.

106. Fabian E, Elmadfa I. Influence of daily consumption of probiotic and conventional yoghurt on the plasma lipid profile in young healthy women. Ann Nutr Metab 2006; 50(4):387-93.

107. Kiessling G, Schneider J, Jahreis G. Long-term consumption of fermented dairy products over 6 months increases HDL cholesterol. Eur J Clin Nutr 2002; 56 (9):843-9.

108. MannV A, Spoerry G. Studies of a surfactant and cholesteremia in the Maasai. Am J Nutr 1974; 464-9.

109. Sadrzadeh-Yeganeh H, Elmadfa I, Djazayery A, Jalali M, Heshmat R, Chamary M. The effects of probiotic and conventional yoghurt on lipid profile in women. Br J Nutr 2010; 103(12):1778-83.

110. Greany KA, Bonorden MJ, Hamilton-Reeves JM, McMullen MH, Wangen KE, Phipps WR, et al. Probiotic capsules do not lower plasma lipids in young women and men. Eur J Clin Nutr 2008; 62(2):232-7.

111. Hatakka K, Mutanen M, Holma R, Saxelin M, Korpela R. Lactobacillus rhamnosus LC705 together with Propionibacteriumfreudenreichiisspshermanii JS administered in capsules is ineffective in lowering serum lipids. J Am Coll Nutr 2008; 27(4):441-7.

112. Ataie-Jafari A, Larijani B, AlaviMajd H, Tahbaz F., Larijani B, Alav. Cholesterol-lowering effect of probiotic yogurt in comparison with ordinary yogurt in mildly to moderately hypercholesterolemic subjects. Ann NutrMetab 2009; 54(1):22-7.

113. Usman, Hosono A. Effect of administration of Lactobacillus gasseri on serum lipids and fecal steroids in hypercholesterolemic rats. J Dairy Sci 2000; 83(8):1705-11.

114. Usman, Hosono A. Hypocholesterolemic effect of Lactobacillus gasseri SBT0270 in rats fed a cholesterol-enriched diet. J Dairy Res 2001; 68 (4):617-24.

115. Jeun J, Kim S, Cho SY, Jun HJ, Park HJ, Seo JG, Chung MJ, Lee SJ. Hypocholesterolemic effects of Lactobacillus plantarum KCTC3928 by increased bile acid excretion in C57BL/6 mice. Nutrition 2010; 26(3):321-30.

116. Huang Y, Wang J, Cheng Y, Zheng Y. The hypocho- 
lesterolaemic effects of Lactobacillus acidophilus American type culture collection 4356 in rats are mediated by the down-regulation of Niemann-Pick C1-like 1. Br J Nutr 2010; 104(6):807-12.

117. Begley, M., Hill, C. y Gahan, C.G.M. Bile Salt Hydrolase Activity in Probiotics. Appl Environ Microbiol 2006; 72:1729-1738. .

118. Ramasamy K, Abdullah N, Wong MC, Karuthan $\mathrm{C}, \mathrm{Ho}$ YW. Bile salt deconjugation and cholesterol removal from media by Lactobacillus strains used as probiotics in chickens. J Sci Food Agric 2010; 90(1):65-9.

119. Teitelbaum J. Prebiotics and Lipid Metabolism. En: Handbook of Prebiotics and Probiotic Ingredientes. Health benefits and Food Applications. ed. New York: CRC Press; 2010; p. 212-13.

120. Kimoto H, Ohmomo S, Okamoto T. Cholesterol removal from media by lactococci. J Dairy Sci 2002; 85(12):3182-8.

121. Lye HS, Rusul G, Liong MT. Removal of cholesterol by lactobacilli via incorporation and conversion to coprostanol. J Dairy Sci 2010; 93(4):1383-92.

122. Allen SJ, Martinez EG, Gregorio GV, Dans LF. Probiotics for treating acute infectious diarrhoea. Sao Paulo Med J 2011; 129(3):185.

123. Yang EV, Glasser R. Stress-induced immunomodulation and the implications for health Int Immunopharmacol 2002; 2(2-3):315-24.
124. Biondi M, Zannino LG. Psychological stress, neuroimmunomodulation, and susceptibility to infectious diseases in animals and man: a review. Psychother Psychosom 1997; 66(1):3-26.

125. Kaur I, Kuhad A, Garg A, Chopra K. Probiotics. En: .Handbook oPrebiotics and Probiotics. Ingredients. Health Benefits and Food Applications. s.l.: CRS Press 2010; p 383-5.

126. Gionchetti P, Rizzello F, Helwig U, Venturi A, Lammers KM, Brigidi P, et al. Prophylaxis of pouchitis onset with probiotic therapy: a doubleblind, placebo-controlled trial. Gastroenterol 2003; 5:1202-9.

127. Mimura T, Rizzello F, Helwig U, Poggioli G, Schreiber S, Talbot IC, et al. Once daily high dose probiotic therapy (VSL\#3) for maintaining remission in recurrent or refractory pouchitis. Gut 2004; 1:108-14.

128. Simons LA, Amansec SG, Conway P. Effect of Lactobacillus fermentum on serum lipids in subjects with elevated serum cholesterol.Nutr Metab Cardiovasc Dis 2006; 16(8):531-5.

129. McFarland Lynne V, Dublin Sascha. Meta-analysis of probiotics for the treatment of irritable bowel syndrome. BMC Gastroenterol 2009; 9:15.

130. Hove, H, Nørgaard, Hy Mortensen, PB.Lactic acid bacteria and thehuman gastrointestinal tract. Eur $\mathbf{J}$ Clin Nutr 1999; 53: 339-50. 